\title{
Onychomycosis with Photodynamic Therapy of Fingernails
}

\author{
Abdur Rafel ${ }^{1 *}$, Ibrahim Sodi ${ }^{1}$, Sattar Said ${ }^{1}$ and Nadima Hussain ${ }^{1}$ \\ 1 Department of Dermatology, Shiraz, Iran. \\ *Corresponding Author : Abdur Rafel, Department of Dermatology, Shiraz,Iran. E-mail: abdur.ra@gmail.com \\ Received date: March 02, 2018;Accepted date : March 22, 2018; Published date: March 27, 2018 \\ Citation : Abdur Rafel, Ibrahim Sodi, Sattar Said and Nadima Hussain, Onychomycosis with Photodynamic Therapy of \\ Fingernails. J .Dermatology and Dermatitis. Doi: 10.31579/2578-8949/028
}

Copyright:@2018 Abdur Rafel. Thisisan open-access article distributed under the terms of The Creative Commons Attribution License, which permits unrestricted use, distribution, and reproduction in any medium, provided the original author and source are credited.

\begin{abstract}
The advances in laser technology have led to many new devices and uses. Lasers have been used to treat onychomycosis, but this technology isinits infancy. Laser therapy for the temporary cosmetic improvementofonychomycosisoftoenails received FDA approval in 2010. We report a patient treating onychomycosis of the fingernails using the Genesis Plus laser. The advantages are the simplicity oftheprocedure, no concernforsystemic (drug) reactions orcontraindications, and theefficacy ofthe procedure. The disadvantages include cost, the necessity for individualization of settings with clinical analysis of nail involvement and thickness, and slight warmth or heat during the procedure. We feel this modality provides a great service to dermatologic patients, especially those with co-morbidities, and dermatologists should be aware of this use.
\end{abstract}

Keywords : onychomycosis; photodynamic therapy .

\section{Introduction}

Laser therapy for the treatment of onychomycosis of the toenails received FDA approval in 2010; however, this technology is still in its infancy. As the population ages, patients require novel treatment approaches to common, annoying, disfiguring entities that are also potential sources of pain due to secondary infections due to trauma and normal daily activities. Fingernail onychomycosis is such a situation. Whether warranted or not, many patients and referring physicians are hesitant to use oral therapies citing drug interactions, metabolism problems, and lack of desire to add another oral medication to a long list of medicaments that are already taken.

We report a patient with onychomycosis of the fingernails who was treated using the Genesis PlusLaser. This is the first time fingernail onychomycosis therapy with lasers has been addressed. Fingernail onychomycosis is visible and distressing for patients of all ages and should not be overlooked, especially in males where cosmesis could be a problem. The advantages of laser treatments are the simplicity of the procedure, no concern for systemic (drug) reactions or contraindications, and the efficacy of the procedure. The disadvantages include cost, the necessity for individualization of settings with clinical analysis of nail involvement and thickness, and slight warmth or heat during the procedure. We feel this modality provides a great service to patients, especially those with comorbidities.

\section{Case Presentation}

This is an 80-year old man who was diagnosed with myasthenia gravis in 2006 and diabetes mellitus in 2010. He takes mestignon, prednisone, prilosac, lasix, novolox, Klor, multivitamins, and warfarin. He has no known allergies. His prior skin history includes eczematous dermatitis, spindle cell tumor, basal cell carcinoma, actinic keratoses, and seborrheic keratoses. He noticed his nails changing in June 2011. On physical exam, there was dystrophy and thinning of the nail plate of the right third and fourth fingernails and the left third fingernail (Figure 1). Fungal cultures were negative and unreliable; therefore, a nail biopsy including PAS stain confirmed the presence of a dermatophyte (Figure 2). The benefits and side effects of current therapies, both topical and oral, were discussed with the patient. It was noted that oral anti-fungal medications are metabolized in the liver and have possibility of side effects and drug interactions.
This patient did not want the risk of oral therapy; therefore, laser therapy was an excellent option with little to no side effects. The laser was set at fluence $16 \mathrm{~J} / \mathrm{cm}^{2}$, pulse duration $0.2 \mathrm{~m} \mathrm{sec}$ and repetition rate $3.0 \mathrm{~Hz}$. Each fingernail received 200 pulses, the thumb received 100 pulses, and the fifth fingernail received 50 pulses. Excellent clinical results were noted after 4 weeks (Figure 3). The patient remains in remission to date (24 months).

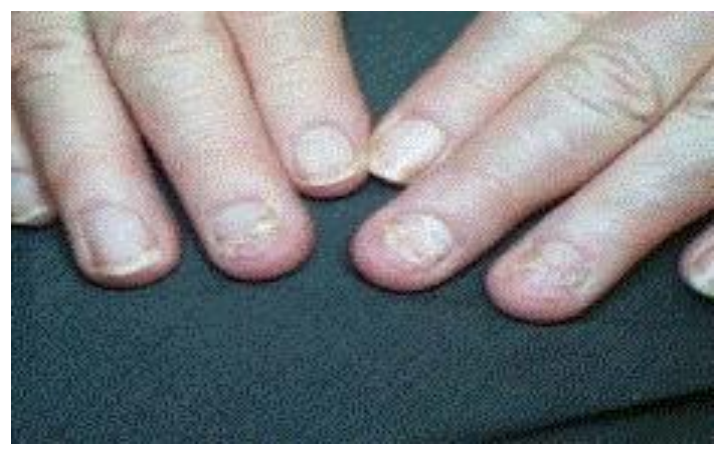

\section{Figure 1}

Nails prior to treatment.

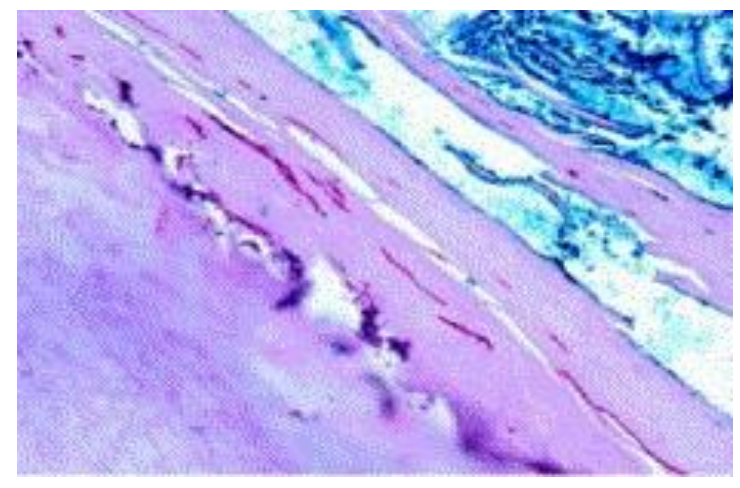

\section{Figure 2}

Dermatophyte identified with PAS stain $60 \mathrm{x}$ resolution. 


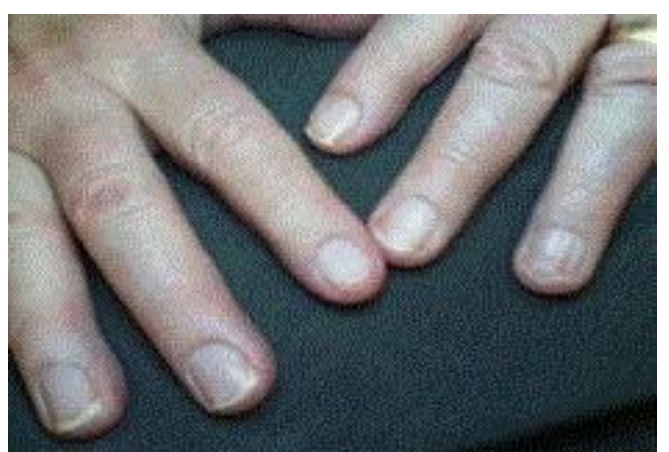

Figure 3

Four weeks post treatment

\section{Discussion}

Onychomycosis is difficult to treat. Topical therapies such as ciclopirox $8 \%$, amoroifine, clotrimazole, and tioconazole have been inadequate and oral medications including terbinafine, itraconazole, and griseofulvin carry a concern about side effects including hepatotoxicity, diarrhea, and rash and cure rates that are less than $100 \%$ [1]. Mechanical debridement can be painful and laborious, and is not adequate if the underlying dermatophytes are not eliminated.

Qiao et al. [2], treated superficial mycosis with photodynamic therapy. Photodynamic therapy requires the application to the area of a topical photosensitizer, usually $20 \%$ levulinic acid, a short-term incubation period, and exposure to a light activating source such as intense pulse light or blue or red light making this a cumbersome therapy [2]. Most of the work and data involving laser and light therapies for onychomycosis appears in the podiatric literature. Heat destroys dermatophytes. Laser energy has been shown to eliminate dermatophytes in vitro. The FDA approved the PinPointe Footlaser, Cutera Genesis Pulse, Q Clear, and Cool Touch7 for the treatment of onychomycosis (Table 1).

Comparison of these studies is difficult since all investigators used their own different criteria for evaluation. Clinical response seems the most useful in evaluation as cultures can be unreliable.

We used a Genesis Plus laser that has been used for clearance of toenail onychomycosis on the fingernails with good results. It is a simple repetitive treatment. Nails should be clean. If the nail is thicker greater than $2 \mathrm{~mm}$, the nail may be debrided. Topical anesthetics and/or blocks are not recommended as the anesthesia interferes with patient feedback. As with all laser treatments, protective eyewear, goggles, must be worn by all persons in the room. The number of treatments vary with each patient and must be individualized depending upon severity of the disease, although this patient responded excellently with one treatment. Fingernails are thinner than toenails, therefore, the number of treatments may be less and responses better [7].
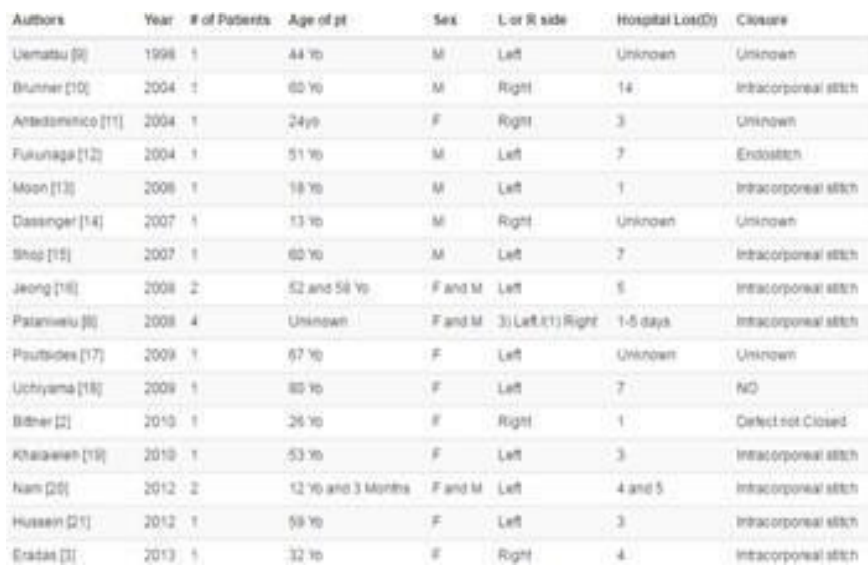

Table 1 Other lasers have been used with the following results.

\section{Conclusion}

Laser therapy is an excellent modality for treating onychomycosis [8] of both fingernails and toenails. It eliminates the potential for drug interactions and side effects that plague older patients. The Genesis Plus gives minimal discomfort and is simple to use. A major problem is cost. The number of treatments must be tailored to each individual patient due to the consideration of the thickness of the nails involved, lifting, and response rate. Although more studies are needed to truly asses recurrence rate, it seems clear this is an efficacious treatment that should be considered as part of the armamentarium for patients who find this infection disturbing, especially with the cosmesis of fingernail involvement [9].

\section{References}

1. Cohen PR, Scher RK. Topical and surgical treatment of onychomycosis. J Am Acad. Dermatol. 1994; 31: 74-75.

2. Quio J, Li R, Ding Y, Fang H. Photodynamic therapy in the treatment of superficial mycoses: an evidence-based evaluation. Mycopathologia. 2010; 170: 339-343.

3. Manevitch Z, Lev D, Hochberg M, Patlhan M, Lewis A, Erick CD. Direct antifungal effect of femtosecond laser on trichophyton rubrum onychomycosis. Photochem Photobiol. 2010; 86: 476-479. doi.org/10.1111/j.1751-1097.2009.00672.x

4. Landsman AS, Robbins AH, Angelini PF, Wu CC, Cook J, Oster M, et al. Treatment of mild, moderate, and severe onychomycosis using 870-and 930nm light exposure. J Am Podiatr Med Assoc. 2010; 100: 166-177

5. Hochman LG. Laser treatment of onychomycosis using a novel 0.65millisecond pulsed Nd:YAG 1064-nm laser. J Cosmet Laser Ther. 2011; 13: 2-5.

6. Gozarev J, Vigintin Z. Nd: YAG laser with temperature control treats onychomycosis effectively. J Laser \& Health Academy. 2010; 1: 1-8.

7. Gupta AK. Device-based therapies for onychomycosis. Skin Therapy Letter. 2012; 17.

8. Cole, Gary. Treating Oncychomycosis with Laser Light. 2016.

9. Scher, Richard. Personal communication. 2016. 